\title{
SOLUTIONS OF THE ONE-DIMENSIONAL POROUS MEDIUM EQUATION ARE DETERMINED BY THEIR FREE BOUNDARY
}

\section{SIGURD ANGENENT}

\section{Introduction}

In this note we present a unique continuation theorem for the free boundary of solutions of the porous medium equation

$$
U_{t}=\left(U^{m}\right)_{x x}
$$

in one space dimension, with $m>1$. As an application of this result we shall also show that solutions whose free boundaries are real analytic arcs, are in fact the positive parts of real analytic functions defined on a neighbourhood of their support.

Our result concerns two weak solutions $U_{1}$ and $U_{2}$ of (1) on a small rectangle

$$
Q=(-a, a) \times(-\tau, \tau)
$$

whose free boundaries are moving. More precisely, we assume that there are two strictly increasing functions $\zeta_{i}:(-\tau, \tau) \rightarrow(-a, a)$ such that

$$
U_{i}(x, t) \begin{cases}>0 & \text { if } x<\zeta_{i}(t), \\ =0 & \text { if } x \geqslant \zeta_{i}(t)\end{cases}
$$

holds for all $(x, t) \in Q$. The $\zeta_{i}(t)$ are called the free boundaries of the respective solutions. It is known that they are $C^{\infty}$ smooth functions (Caffarelli and Friedmann first showed that they are $C^{1}$, and using this Aronson and Vazquez, Kreis and Höllig, and the author proved independently and by different means that the boundaries are indeed $C^{\infty}$ ).

Under special circumstances the free boundary may even be real analytic, but in general this need not be true.

Our main result is the following.

THEOREM 1.1. If $\zeta_{1}(0)=\zeta_{2}(0)$, then either the two solutions $U_{1}$ and $U_{2}$ coincide for $t \leqslant 0$, or else there is $k>0$ such that

$$
\zeta_{1}^{(k)}(0) \neq \zeta_{2}^{(k)}(0)
$$

In particular, if the two solutions are different, then their free boundaries are different.

It may happen that the two free boundaries coincide for $t \leqslant 0$. One can construct many examples of $U_{i}$ with this property, by letting $U_{i}$ be the weak solution of (1) on the closure of $Q$, with boundary condition $U_{i}(-a, t)=f_{i}(t)$ and initial condition $U_{i}(x,-\tau)=g(x)$, where the $f_{i}(t)$ and $g(x)$ are continuous functions with

$$
f_{1}(-\tau)=f_{2}(-\tau)=g(-a)
$$

\section{Received 3 April 1989.}

1980 Mathematics Subject Classification (1985 Revision) 35K15.

While I was working on this paper, I was partially supported by National Science Foundation grant DMS-8801486, and Air Force Office of Scientific Research grant AFOSR-87-0202. 
and $g(x) \equiv 0$ for $x \geqslant 0$. Then, if the $f_{i}$ and $g$ are small enough, the weak solutions will exist on the entire rectangle $Q$, and their free boundaries will not reach the right edge $\{a\} \times(-\tau, \tau)$. If one chooses the boundary values $f_{1,2}$ so that they coincide for $t \leqslant 0$, but not for $t>0$ (for example, $f_{1}(t)<f_{2}(t)$ when $t>0$ ), then the uniqueness of the weak solution implies that the $U_{i}$ and hence their free boundaries $\zeta_{i}(t)$ must coincide for $t \leqslant 0$. Our result implies that they must be different for $t>0$.

This example also shows that the free boundaries do not have to be real analytic functions, even though they are always $C^{\infty}$ smooth.

We shall actually prove a slightly more general result, since we shall consider two radially symmetric solutions of the porous medium equation in $\mathbb{R}^{n}$. My original motive for looking at this problem came from a question of P. E. Souganidis, who asked whether the free boundary of such a radially symmetric solution determines the entire solution (the answer is 'yes').

In the proof of Theorem 1.1 we compute a 'normal form' for a typical intersection of the two free boundaries $\zeta_{i}(t)$. As a consequence of this analysis, we get an analogue of Matano's lap-number result [11]. The result is about two solutions $U_{i}(x, t)$ both of which are assumed to be continuous on the closure of $Q$. Let $\mathscr{U}_{1}(t)$ and $\mathscr{U}_{2}(t)$ be the graphs of their positive parts,

$$
\mathscr{U}_{i}(t)=\left\{(x, U(x, t)):-a<x \leqslant \zeta_{i}(t)\right\}
$$

THEOREM 1.2. If $U_{1}(-a, t) \neq U_{2}(-a, t)$ for all $t \in(-\tau, \tau)$, then at each time $t$ the two curves $\mathscr{U}_{i}(t)$ have at most a finite number of intersections, and this number does not increase with time. In fact, whenever the two free boundaries meet, the number of intersections drops.

In the last two sections we use the unique continuation theorem (1.1) to prove the analyticity of certain solutions of the porous medium equation.

THEOREM 1.3. Let $U$ be a solution of (1) whose free boundary is moving. If the free boundary is real analytic, then $U(x, t)^{m-1}$ can be extended across the free boundary as a real analytic function; that is, near $(0,0)$ one can write the positive part of $U$ as a convergent power series

$$
U(x, t)^{m-1}=\sum_{k, l \geqslant 0} c_{k l} x^{k} t^{l} \quad(x \leqslant \zeta(t)) .
$$

In the proof we use a Cauchy-Kovalevski kind of construction to show that there is such a power-series solution with the same free boundary as our given solution $U$, and then we conclude from Theorem 1.1 that this power series must coincide with $U$.

Combined with the result in [2] this implies the following analyticity result for solutions of the porous medium equation.

THEOREM 1.4. Let $U \in C(\mathbb{R} \times[0, T])$ be a weak solution of (1), whose support is of the form

$$
\operatorname{supp} U=\left\{(x, t) \in \mathbb{R} \times[0, T]: \zeta_{-}(t) \leqslant x \leqslant \zeta_{+}(t)\right\},
$$

where $\zeta_{ \pm}(t)$ are strictly monotone functions. Assume in addition that $U(x, t)>0$ for $\zeta_{-}(t)<x<\zeta_{+}(t)$. Then $U^{m-1}$ is the positive part of a real analytic function on an open neighbourhood of $\operatorname{supp} U$ in $\mathbb{R} \times(0, T]$. 
Indeed, under the hypotheses of this theorem it is shown in [2] that both free boundaries $\zeta_{ \pm}$are real analytic curves, for $t>0$, so that Theorem 1.4 follows immediately from Theorem 1.3.

\section{Radially symmetric solutions}

Let $U_{1,2}$ be two solutions of the porous medium equation,

$$
U_{t}=\Delta\left(U^{m}\right)
$$

defined on $\Omega \times(-\tau, \tau)$, where $\Omega$ is the annulus

$$
\Omega=\left\{x \in \mathbb{R}^{n}: a<|x|<b\right\} .
$$

We shall assume that there are strictly decreasing functions $\zeta_{i}:(-\tau, \tau) \rightarrow(a, b)$ such that

$$
U_{i}(x, t) \begin{cases}=0 & \text { if }|x| \leqslant \zeta_{i}(t) \\ >0 & \text { if }|x|>\zeta_{i}(t)\end{cases}
$$

holds for $-\tau<t<\tau$. Then one can adapt the arguments of Caffarelli and Friedman [6] to prove that the $\zeta_{i}(t)$ are $C^{1}$ functions of time.

Instead of working with the $U_{i}$, we shall consider $u_{i}:=U_{i}^{m-1}$, the so-called 'pressure' related to the 'density' $U_{i}$. These functions satisfy the 'pressure equation'

$$
u_{t}=u \Delta u+\gamma(\nabla u)^{2}
$$

in which $\gamma=(m-1)^{-1}$. For radially symmetric functions this equation is equivalent to

$$
u_{t}=u\left(u_{r r}+\frac{n-1}{r} u_{r}\right)+\gamma\left(u_{r}\right)^{2}
$$

At the free boundary we have $u_{i, r}\left(\zeta_{i}(t), t\right)>0$, so that we can introduce two new functions $r_{i}(y, t)$, which are related to the $u_{i}$ via

$$
u_{i}\left(r_{i}, t\right)=\frac{1}{2} y^{2} .
$$

Since the $u_{i}$ are $C^{1}$ functions for $r \geqslant \zeta_{i}(t)$, there will be a small $y_{*}>0$ such that both $r_{i}$ are defined for $|y| \leqslant y_{*}$. They will be even functions of $y$.

If we differentiate the defining equation a couple of times, then we get the relations

$$
\begin{aligned}
u_{t}+u_{r} r_{t} & =0, \\
u_{r} r_{y} & =y, \\
u_{r r}\left(r_{y}\right)^{2}+u_{r} r_{y y} & =0 .
\end{aligned}
$$

Upon substitution of these relations in (2), one finds that

$$
r_{t}=\frac{y^{2}}{2 r_{y}^{2}} r_{y y}-\left(\gamma+\frac{1}{2}\right) \frac{y}{r_{y}}-\frac{(n-1) y^{2}}{2 r} .
$$

This equation has the form

where $f$ is the function

$$
r_{t}=f\left(y, r, \frac{r_{y}}{y}, r_{y y}\right)
$$

$$
f(y, R, P, Q)=\frac{Q}{2 P^{2}}-\frac{\gamma+\frac{1}{2}}{P}-\frac{(n-1) y^{2}}{2 R} .
$$


A short computation then shows that

and

$$
f_{Q}>0
$$

$$
f_{P}(0, R, P, P)=(2 \gamma-1) f_{Q}(0, R, P, P) .
$$

In addition to (4) the function $r$ also satisfies the following boundary condition:

$$
r_{y}(0, t)=0 \text {. }
$$

The quantity $p=u_{r}=y / r_{y}=1 / P$ satisfies the equation

$$
p_{t}=\frac{p^{2}}{2}\left(p_{y y}+(2 \gamma+1) \frac{p_{y}}{y}\right)+(n-1)\left[p^{2} / r-y^{2} p / 2\right] \text {. }
$$

Since the Caffarelli-Friedmann result extends to the radially symmetric case, we have continuity of $p$. Then, as in [2] we get Hölder continuity of $p$, and from that we get $C^{\infty}$ smoothness of $p$, again, as in [2].

\section{Normal form near an intersection}

Let $r_{1}(y, t), r_{2}(y, t)$ be the two solutions of (4) which correspond to the two solutions $U_{i}$ of the porous medium equation with which we started. We have assumed that the two free boundaries $\zeta_{i}(t)$ meet each other at $t=0$, and this implies that

$$
r_{1}(0,0)=r_{2}(0,0) \text {. }
$$

Since the $r_{i}$ are smooth even functions, they also satisfy the boundary condition

for $t \in(-\tau, \tau)$.

$$
r_{1, y}(0, t)=r_{2, y}(0, t)=0
$$

Both $r_{i}$ are solutions of the same equation (4), so that their difference $v=r_{1}-r_{2}$ will satisfy a linear parabolic equation in which the first-order term turns out to be singular:

$$
v_{t}=a(y, t) v_{y y}+\frac{b(y, t)}{y} v_{y}+c(y, t) v
$$

The coefficients $a, b$ and $c$ are given by

$$
\begin{aligned}
& a(y, t)=\int_{0}^{1} f_{Q}\left(y, r^{\theta}(y, t), \frac{r_{y}^{\theta}(y, t)}{y}, r_{y y}^{\theta}(y, t)\right) d \theta, \\
& b(y, t)=\int_{0}^{1} f_{P}\left(y, r^{\theta}(y, t), \frac{r_{y}^{\theta}(y, t)}{y}, r_{y y}^{\theta}(y, t)\right) d \theta, \\
& c(y, t)=\int_{0}^{1} f_{R}\left(y, r^{\theta}(y, t), \frac{r_{y}^{\theta}(y, t)}{y}, r_{y y}^{\theta}(y, t)\right) d \theta,
\end{aligned}
$$

in which $r^{\theta}=(1-\theta) r_{1}+\theta r_{2}$. At $y=0$ the first two coefficients satisfy the relation

$$
b(0, t)=(2 \gamma-1) a(0, t),
$$

which follows from (5) and the fact that, by l'Hôpital's rule,

$$
\lim _{y \rightarrow 0} \frac{r_{y}(y, t)}{y}=r_{y y}(0, t)
$$


By a smooth change of coordinates we can make the first coefficient a constant. Just define

$$
x(y, t)=\int_{0}^{y} \frac{d \eta}{\sqrt{ }(a(\eta, t))} .
$$

Then the function $w$, defined by $w(x(y, t), t)=v(y, t)$, satisfies

$$
w_{t}=w_{x x}+\frac{B(x, t)}{x} w_{x}+C(x, t) w
$$

where $B, C$ are smooth functions of $(x, t)$, which are even in $x$. Moreover $B(0, t)$ is given by

$$
B(0, t)=\frac{b(0, t)}{a(0, t)}=2 \gamma-1 .
$$

Next, if we choose a positive smooth even function $\varphi$ which satisfies

$$
\varphi_{x}(x, t)=\frac{B(x, t)-B(0, t)}{2 x}
$$

and $\varphi(0, t)=1$, then the function $\hat{w}(x, t)=w(x, t) / \varphi(x, t)$ satisfies

$$
\hat{w}_{t}=\hat{w}_{x x}+\frac{2 \gamma-1}{x} \hat{w}_{x}+D(x, t) \hat{w}
$$

for some smooth function $D(x, t)$ which is even in $x$. In addition, we have

$$
\hat{w}_{x}(0, t)=0 \quad(t \in(-\tau, \tau)) .
$$

As in $[4,12]$ we can compute what the highest-order terms of the Taylor expansion of $\hat{w}$ look like. We have assumed that at $t=0$ one has $\hat{w}(0,0)=0$. In addition to this we now assume that $\hat{w}(x, 0)$ has an $m$ th-order zero as a function of $x$, at $x=0$. In other words, we assume that

$$
\hat{w}(x, 0)=c x^{m}+O\left(x^{m+1}\right) .
$$

Since $\hat{w}$ is an even function, $m=2 n$ must be even, and one can replace $O\left(x^{m+1}\right)$ by $O\left(x^{m+2}\right)$.

By differentiating (9) repeatedly with respect to time, one proves by induction that

$$
\frac{\partial^{k} \hat{w}}{\partial t^{k}}(x, 0)=c 2^{2 k} \frac{n !}{(n-k) !} \frac{\Gamma(n+\gamma)}{\Gamma(n-k+\gamma)} x^{2(n-k)}+\ldots
$$

and thus

$$
\hat{w}(x, t)=c \sum_{k=0}^{n} \frac{\Gamma(n+\gamma)}{\Gamma(n-k+\gamma)} \frac{n !}{(n-k) !} x^{2(n-k)} \frac{(4 t)^{k}}{k !}+\ldots,
$$

which can also be written as

$$
\begin{aligned}
\hat{w}(x, t) & =c(4 t)^{n} \sum_{k=0}^{n}\left(\begin{array}{l}
n \\
k
\end{array}\right) \frac{\Gamma(n+\gamma)}{\Gamma(k+\gamma)}\left(\frac{x^{2}}{4 t}\right)^{k}+\ldots \\
& =n ! c(4 t)^{n} L_{n}^{\gamma-1}\left(\frac{-x^{2}}{4 t}\right)+\ldots,
\end{aligned}
$$

where $L_{n}^{\gamma-1}(x)$ is the $n$ th-degree Laguerre polynomial of order $\gamma-1$ (see [13, p. 101]). 


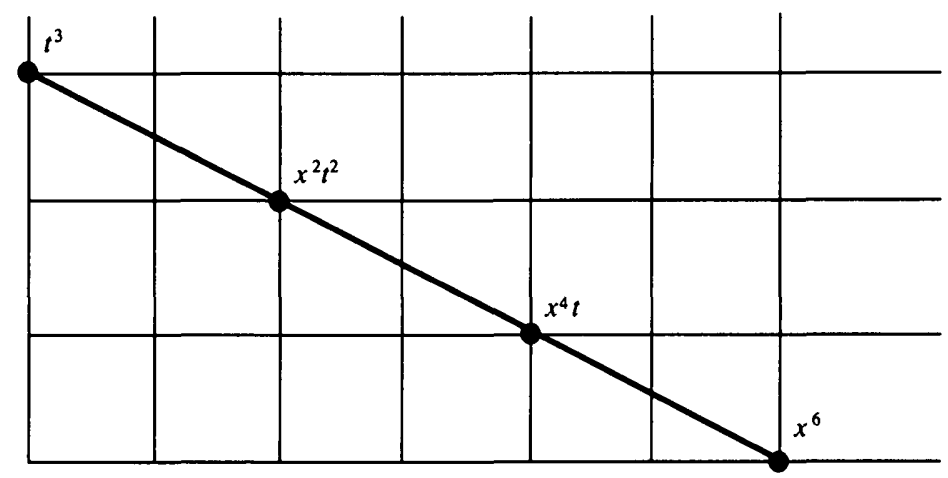

FIGURE 1. The Newton polygon when $m=6$

In particular one sees from this that

$$
\hat{w}(0, t)=c^{\prime} t^{n}+\ldots,
$$

so that near $t=0$ there is no other $t$ at which the two free boundaries will intersect.

From this one can also compute the Newton polygon of $\hat{w}$ at $\left(0, t_{0}\right)$, that is, the convex hull of all pairs $(k, l) \in \mathbb{N} \times \mathbb{N}$ for which the coefficient of $x^{k} t^{l}$ in the Taylor expansion of $\hat{w}$ does not vanish. It consists of the straight line through $(n, 0)$ and $(0,2 n)$, and parts of the two coordinate axes.

Up to a numerical factor the Laguerre polynomials $L_{n}^{\gamma-1}(x)$ are obtained by orthogonalizing $\left\{1, x, x^{2}, x^{3}, \ldots\right\}$ in $L_{2}\left(\mathbb{B}_{+}, x^{\gamma-1} e^{-x} d x\right)$. This implies, as is well known, that $L_{n}^{\gamma-1}(x)$ has $n$ distinct zeros on the positive real line [13].

If $\hat{w}$ were equal to the highest-order part of its Taylor series, then its zero set would consist of $n$ parabolae of the form $4 \vartheta_{j} t=-x^{2}(j=1, \ldots, n)$, where the $\vartheta_{j}$ are the zeros of $L_{n}^{\gamma-1}(\vartheta)$.

Using the Malgrange preparation theorem [8] and the simplicity of the zeros of the Laguerre polynomials, one proves that the zero set of $\hat{w}$ near $(0,0)$ consists of $n$ $C^{\infty}$-smooth curves, each of which is a graph of the form

$$
t=-T_{j}(x), \quad 1 \leqslant j \leqslant n,
$$

where $T_{j}$ is a smooth function which satisfies

$$
T_{i}(0)=T_{i}^{\prime}(0)=0 \quad \text { and } \quad T_{i}^{\prime \prime}(0)=1 / 2 \vartheta_{j} .
$$

This means that in a small neighbourhood of the origin, the function $\hat{w}(\cdot, t)$ has $n$ zeros for $t<0$, and no zeros for $t>0$.

Consider the two curves $\mathscr{U}_{i}(t)$ of the introduction, and assume that the two solutions $u_{i}(x, t)$ of the porous medium equation that define them are different. It follows from the regularity results for parabolic partial differential equations in [7] that they are real analytic curves away from their end points $\left(\zeta_{t}(t), t\right)$. If they should have an infinite number of intersections at some time $t_{0}$, these intersections must therefore accumulate at the end points, and these end points must coincide; that is, $\zeta_{1}\left(t_{0}\right)=\zeta_{2}\left(t_{0}\right)$. In the next section we shall show that $\partial_{x}^{2 k} \hat{w}\left(0, t_{0}\right) \neq 0$ for some integer $k>0$, so that $\hat{w}\left(x, t_{0}\right) \neq 0$ for small $x \neq 0$, and two curves $\mathscr{U}_{i}\left(t_{0}\right)$ do not intersect near $\left(\zeta_{i}\left(t_{0}\right), t_{0}\right)$, except at the point $\left(\zeta_{i}\left(t_{0}\right), t_{0}\right)$ itself.

Thus, assuming the 'non-flatness' result of the next section, we have shown that the curves $\mathscr{U}_{1}(t)$ and $\mathscr{U}_{2}(t)$ have at most a finite number of intersections. 
If this number of intersections changes at some time $t_{0}$, then the $\mathscr{U}_{i}\left(t_{0}\right)$ either have a non-transversal intersection, or their end points coincide. In the first case it follows from the results in any of the references $[12,11,4,3]$ that the number of intersections must decrease.

The second case occurs exactly when $\hat{w}\left(0, t_{0}\right)=0$, and since the zeros of $\hat{w}\left(\cdot, t_{0}\right)$ correspond to intersections of $\mathscr{U}_{1}\left(t_{0}\right)$ and $\mathscr{U}_{2}\left(t_{0}\right)$, we have just shown that the number of zeros must decrease in this situation as well.

This completes the proof of Theorem 1.2, provided we show that $\hat{w}\left(\cdot, t_{0}\right)$ cannot have a zero of infinite order, unless it vanishes identically. We do this in the next section, and in the proof we shall use the following lemma, which follows from what we have just done.

LEMMA 3.1. If $y \rightarrow \hat{w}\left(y, t_{0}\right)$ has a zero of order $m=2 n$, then

$$
\lim _{\tau \rightarrow \infty} e^{2 n \tau} \hat{w}\left(e^{-\tau} \xi, t_{0}-e^{-2 \tau}\right)=c L_{n}^{\gamma-1}\left(\xi^{2} / 4\right)
$$

for some constant $c$. If $y \rightarrow \hat{w}\left(y, t_{0}\right)$ has a zero of infinite order, then $c=0$, that is, the limit vanishes for any $n$.

\section{Non-flatness}

In this section we use some of the ideas in [3] to prove that $\hat{w}$ cannot have a zero of infinite order.

First, as in [3], we note that we can assume that the solution $\hat{w}$ is defined on all $\mathbb{R} \times[0, T]$. Indeed, if $\hat{w}\left(x_{0}, t_{0}\right) \neq 0$ for some $\left(x_{0}, t_{0}\right)$, then $\hat{w}$ is non-zero on a neighbourhood of this point, say on $\left(x_{0}-\varepsilon, x_{0}+\varepsilon\right) \times\left(t_{0}-\varepsilon, t_{0}+\varepsilon\right)$. Then we can change $\hat{w}$ on the region $x \geqslant x_{0}$ so that $\hat{w}$ becomes defined on the entire strip $\mathbb{R} \times\left(t_{0}-\varepsilon, t_{0}+\varepsilon\right)$, and does not vanish when $|x| \geqslant x_{0}$.

The new $\hat{w}$ also satisfies an equation like (9), since the old and the new $\hat{w}$ coincide for $|x| \leqslant x_{0}$, while the new $\hat{w}$ does not vanish for larger $x$. Therefore we can just define $D(x, t)$ to be $\left(\hat{w}_{t}-\hat{w}_{x x}-(2 \gamma-1) \hat{w}_{x} / x\right) / \hat{w}$. Clearly we could have chosen $\hat{w}$ to have bounded derivatives, so that the $D$ we get is a bounded function on $\mathbb{R} \times\left(t_{0}-\varepsilon, t_{0}+\varepsilon\right)$.

Introduce new coordinates $\xi$ and $\tau$, given by

$$
t=t_{0}-e^{-2 \tau}, \quad x=e^{-\tau} \xi .
$$

Then $\hat{w}$ as a function of $\xi, \tau$ satisfies

$$
\hat{w}_{\tau}=2 \hat{w}_{\xi \xi}-\xi \hat{w}_{\xi}+\frac{4 \gamma-2}{\xi} \hat{w}_{\xi}+2 e^{-2 \tau} D\left(e^{-\tau} \xi, t_{0}-e^{-2 \xi}\right) \hat{w}
$$

so that $z=e^{-\frac{1}{8} \xi^{2}} \hat{w}\left(e^{-\tau} \xi, t_{0}-e^{-2 \tau}\right)$ satisfies

where $\mathscr{H}_{\gamma}$ is the operator

$$
z_{\tau}=\left(-\mathscr{H}_{\gamma}+P(t)\right) z
$$

$$
\begin{aligned}
-\mathscr{H}_{\gamma} & =2 e^{-\frac{1}{8} \xi^{2}}\left[\left(\frac{\partial}{\partial \xi}\right)^{2}-\frac{\xi}{2} \frac{\partial}{\partial \xi}+\frac{2 \gamma-1}{\xi} \frac{\partial}{\partial \xi}\right] e^{\frac{1}{\xi^{2}}} \\
& =2\left(\frac{\partial}{\partial \xi}\right)^{2}+\frac{4 \gamma-2}{\xi} \frac{\partial}{\partial \xi}+\left(\gamma-\frac{1}{8} \xi^{2}\right)
\end{aligned}
$$

and $P(t)$ is given by

$$
P(t) z=2 e^{-2 t} D\left(t_{0}-e^{-2 \xi}, e^{-\tau} \xi\right) z .
$$


The operator $\mathscr{H}_{y}$ defines an unbounded operator in the Hilbert space

with domain

$$
E=\left\{z \in L_{2}\left(\mathbb{R},|\xi|^{2 \gamma-1} d \xi\right): z(\xi) \equiv z(-\xi)\right\}
$$

$$
\operatorname{dom}\left(\mathscr{H}_{y}\right)=\{\varphi \in \mathscr{S}(\mathbb{R}): \varphi(\xi) \equiv \varphi(-\xi)\},
$$

where $\mathscr{S}(\mathbb{R})$ is the Schwartz space of rapidly decreasing functions. For integer values of $\gamma$ the operator $\mathscr{H}_{y}$ is the radial part of the quantum-mechanical Hamiltonian for the $\gamma$-dimensional harmonic oscillator, in polar coordinates.

LEMMA 4.1. The operator $\mathscr{H}_{\gamma}$ is symmetric and positive semidefinite. Its closure $H_{\gamma}$ is self-adjoint.

Proof. Integration by parts shows that, for $\varphi, \psi \in \operatorname{dom}\left(\mathscr{H}_{\nu}\right)$ one has

$$
\left(\varphi, \mathscr{H}_{\gamma} \psi\right)=\int_{0}^{\infty} \xi^{2 \gamma-1}\left\{\overline{\varphi^{\prime}(\xi)} \psi^{\prime}(\xi)+\left(\frac{1}{8} \xi^{2}-\gamma\right) \overline{\varphi(\xi)} \psi(\xi)\right\} d \xi
$$

which shows that $\mathscr{H}_{y}$ is symmetric and bounded from below. By inspection one sees that the functions

$$
\psi_{n}(\xi)=L_{n}^{\gamma-1}\left(\frac{1}{4} \xi^{2}\right) e^{-\frac{1}{8} \xi^{2}}
$$

are eigenfunctions of $\mathscr{H}_{y}$, with eigenvalue $-2 n$.

Since the $\psi_{n}(\xi)$ form a complete orthonormal system in $E$ the operator $\mathscr{H}_{y}$ has exactly one self-adjoint extension, namely its closure. The spectrum of $H_{y}$ consists of the non-negative even integers, so that $H_{y}$ is positive semidefinite.

Thus the gaps in the spectrum of $H_{\gamma}$ all have length 2, while the operator $P(t)$ tends to zero (in operator norm on $E$ ). We can therefore apply [1, Lemma 6], which says that, for some integer $k \geqslant 0$ and some constant $c \neq 0$,

in the $E$ norm.

$$
\lim _{\tau \rightarrow \infty} e^{2 k \tau} z(\xi, \tau)=c \psi_{k}(\xi)
$$

If the solution $\hat{w}\left(x, t_{0}\right)$ had a zero of infinite order, then, by Lemma (3.1), this would imply that

$$
\lim _{\tau \rightarrow \infty} e^{2 k \tau} z(\xi, \tau)=0
$$

pointwise, on $\mathbb{R}_{+}$, which clearly contradicts (10). Therefore $\hat{w}$ cannot have a zero of infinite order, and we have completed the proof of Theorems 1.1 and 1.2.

\section{Analyticity across the boundary}

We turn to the proof of Theorem 1.3.

Let $\zeta:(-\tau, \tau) \rightarrow(-a, a)$ be a real analytic function, with $\zeta^{\prime}(t)>0$. Then we shall show that there exists a solution $u$ of

of the form

$$
u_{t}=u u_{x x}+\gamma\left(u_{x}\right)^{2}
$$

$$
u(x, t)=\sum_{n=1}^{\infty} u_{n}(t)(x-\zeta(t))^{n},
$$

where the $u_{n}(t)$ are real analytic functions, and for every $\delta>0$ there is $\rho_{\delta}>0$ such the series (11) converges uniformly for $|x| \leqslant \rho_{\delta},|t| \leqslant \tau-\delta$. 
If $\hat{u}(x, t)$ is defined to be $u(x, t)$ for $x<\zeta(t)$, and 0 for $x \geqslant \zeta(t)$, then $\hat{u}$ is a weak solution of the porous medium equation, which is defined on an open neighbourhood of the prescribed free boundary $x=\zeta(t)$.

By Theorem 1.1 there is at most one solution of the form (11), and if such a solution exists, then there are no other $C^{\infty}$ solutions; that is, any $C^{\infty}$-smooth solution with free boundary $x=\zeta(t)$ must coincide with the given solution (11).

Assuming that such a solution exists, one must have

$$
u_{1}(t)=u_{x}(\zeta(t), t)=-\zeta^{\prime}(t) / \gamma
$$

by the well-known formula for the speed of the free boundary of a solution of the porous medium equation. One can substitute the ansatz (11) in the equation (1), and by raking together terms which contain the same power of $(x-\zeta(t))$, one obtains a recurrence relation for the coefficients $u_{n}(t)$, from which they can be computed, one at a time.

Instead of computing the coefficients $u_{n}(t)$ and proving directly that the series in (11) converges, we shall go back to the transformation

and consider the function

$$
\begin{gathered}
u(x(y, t), t)=\frac{1}{2} y^{2} \\
p(y, t)=u_{x}(x(y, t), t) .
\end{gathered}
$$

In Section 2 we noted that the porous medium equation for $u$ is equivalent to the following equation for $p$ :

$$
\left.\begin{array}{l}
p_{y y}+\frac{2 \gamma+1}{y} p_{y}=-2 \frac{p_{t}}{p^{2}}, \\
p_{y}(0, t)=0, \quad p(0, t)=-\zeta^{\prime}(t) / \gamma .
\end{array}\right\}
$$

We shall prove that this problem has a solution of the form

$$
p(y, t)=\sum_{k=0}^{\infty} p_{k}(t) y^{2 k}
$$

for certain real analytic functions $p_{k}(t)$.

Given such a solution, $P(u, t)=p(\sqrt{ } u, t)$ is a well-defined holomorphic function, and one can recover $u(x, t)$ from $p$ by solving

$$
\partial v / \partial x=P(v(x, t), t), \quad v(0, t)=0
$$

and putting $u(x, t)=v(x-\zeta(t), t)$. If $p$ is analytic, then $u$ will be analytic also.

Therefore, to prove that $u$ exists, we must solve (12). In Theorem 6.1 we shall show that for every $t_{0} \in(-\tau, \tau)$ there is a real analytic solution $p^{t_{0}}(y, t)$ on some rectangle

$$
R^{t_{0}}=\left\{(y, t):|y|<\delta\left(t_{0}\right),\left|t-t_{0}\right|<\delta\left(t_{0}\right)\right\} .
$$

By the uniqueness of the real analytic solution, these $p^{t_{0}}$ coincide on the overlaps of the squares $R^{t_{0}}$, and one can piece them together to form a solution $p$ on some open neighbourhood of $(-\tau, \tau) \times\{0\}$ in $(-\tau, \tau) \times(-a, a)$. Moreover, if $p(y, t)$ is a solution then so is $p(-y, t)$, so that the uniqueness of the solution also implies that it is an even function of the $y$ variable. Theorem 1.3 therefore follows from Theorem 6.1.

\section{A Cauchy-Kovalevski theorem}

Instead of dealing with power series, we shall consider holomorphic functions of the two complex variables $y$ and $t$. We can do this since such functions can be written as a power series, and any power series yields such a holomorphic function. 
If $r_{1}, \ldots, r_{N}$ are positive then we let $D\left(r_{1}, \ldots, r_{N}\right)$ denote the open polydisk

$$
D\left(r_{1}, \ldots, r_{N}\right)=\left\{\left(z_{1}, \ldots, z_{N}\right) \in \mathbb{C}^{N}:\left|z_{j}\right|<r_{j}\right\} .
$$

Let $\mathscr{A}_{\alpha}$ be the differential operator

$$
\mathscr{A}_{\alpha}=\left(\frac{\partial}{\partial y}\right)^{2}+\frac{2 \alpha-1}{y} \frac{\partial}{\partial y}
$$

where $\alpha \in \mathbb{C}$ is a constant with $\operatorname{Re}(\alpha)>0$.

THEOREM 6.1. Let $f(u, v, y, t)$ be a holomorphic function on a neighbourhood of the origin in $\mathbb{C}^{4}$. Then for any holomorphic function $\varphi(t)$ on a neighbourhood of the origin in $\mathbb{C}$, such that $f\left(\varphi(0), \varphi^{\prime}(0), 0,0\right)$ is defined, there is a holomorphic solution of

$$
\left.\begin{array}{rl}
\mathscr{A}_{\alpha}(u) & =f\left(u, u_{t}, y, t\right), \\
u(0, t) & =\varphi(t), \\
u_{y}(0, t) & =0
\end{array}\right\}
$$

on the polydisk $D(a, \tau)$, for some small enough $a, \tau>0$.

Since the operator $\mathscr{A}_{\alpha}$ has a regular singularity at $y=0$, it seems proper to call (13) a 'regular-singular Cauchy problem'.

We first prove a linear inhomogeneous version of this theorem, and then use Newton's method to solve the nonlinear problem.

THEOREM 6.2. Let $P, Q$ and $f$ be bounded holomorphic functions on the polydisk $D(a, \tau)$. Then the linear inhomogeneous Cauchy problem

$$
\left.\begin{array}{rl}
\mathscr{A}_{\alpha}(u) & =P(y, t) u_{t}+Q(y, t) u+f(y, t), \\
u(0, t) & =0, \\
u_{y}(0, t) & =0
\end{array}\right\}
$$

has a holomorphic solution $u$ on $D(a, \tau)$. This solution satisfies

$$
|u(y, t)|+\left|u_{t}(y, t)\right| \leqslant c\|f\||y|^{2} \exp \left(\frac{c K|y|^{2}}{\tau-|t|}\right),
$$

in which the constant $c$ only depends on $\alpha$ and $\tau$. The constant $K$ is given by

$$
K=\|P\|+\|Q\| \text {. }
$$

The norm $\|\cdot\|$ which we have used is the supremum norm on $D(a, \tau)$ :

$$
\|f\|=\sup _{(y, t) \in D(a, \tau)}|f(y, t)| .
$$

This is a variation on the usual linear Cauchy-Kovalevski theorem, as it can be found in partial differential equation textbooks like F. John's [10]. The conclusion of our theorem is slightly stronger, since one usually only gets a solution on a pyramidlike region of the form $|t|<\tau-C|y|$ for some constant $C$. Instead, we get a solution on the whole polydisk $D(a, \tau)$. This phenomenon is related to the infinite speed of propagation associated with parabolic partial differential equations; if we had 
included a term with a second-order derivative in the right-hand side of (14), then the equation would have been hyperbolic in nature, and our solution would only have been defined on the smaller pyramidal region.

Proof. Our strategy is the following: we invert the operator $\mathscr{A}_{\alpha}$, and write (14) as an integral equation of the type $u=T_{\alpha}\left[P u_{t}+Q u+f\right]$, which we solve by a simple Picard iteration.

The unique solution $u$ of

$$
\mathscr{A}_{\alpha}(u)=f(y, t), \quad u(0, t)=u_{y}(0, t)=0
$$

is easily found by multiplying the equation by $y^{2 \alpha-1}$, and integrating twice. It is given by

$$
u(y, t)=\left(T_{\alpha} f\right)(y, t)=y^{2} \int_{0}^{1} \int_{0}^{\lambda}(\mu / \lambda)^{2 \alpha-1} f(\mu y, t) d \mu d \lambda .
$$

Thus the inverse of $\mathscr{A}_{\alpha}$ is given by $T_{\alpha}$. A straightforward computation shows the following.

LEMMA 6.3. If $f(y, t)$ is any measurable function on the polydisk $D(a, \tau)$, such that $|f(y, t)| \leqslant C(t)|y|^{2 n}$ for $|y|<a,|t|<\tau$, and some constant $n \geqslant 0$, then

holds on $D(a, \tau)$.

$$
\left|\left(T_{\alpha} f\right)(y, t)\right| \leqslant \frac{C(t)}{4(n+1)(n+\operatorname{Re}(\alpha))}|y|^{2(n+1)}
$$

Thus our linear regular-singular Cauchy problem (14) is equivalent to

$$
u=T_{\alpha}\left(P u_{t}+Q u+f\right)
$$

Inductively we define $u^{0}(y, t)=0$, and

$$
u^{n+1}=T_{\alpha}\left(P u_{t}^{n}+Q u^{n}+f\right) .
$$

The $u^{n}$ are clearly well defined on $D(a, \tau)$. We show that they converge uniformly on compact subsets of $D(a, \tau)$. Recall that

$$
K=\max \{|P(y, t)|,|Q(y, t)|:|y| \leqslant a,|t| \leqslant \tau\} .
$$

Then $v^{n}=u^{n+1}-u^{n}$ with $n \geqslant 2$ satisfies

while $v^{1}=u^{1}$ so that

$$
\left|v^{n}(y, t)\right| \leqslant K T_{\operatorname{Re}(\alpha)}\left(\left|v^{n}\right|+\left|v_{t}^{n}\right|\right),
$$

$$
\left|v^{1}(y, t)\right| \leqslant T_{\operatorname{Re}(\alpha)}|f(y, t)| \leqslant \frac{\|f\|_{\infty}}{4(\operatorname{Re}(\alpha)+1)}|y|^{2} .
$$

We can estimate $v_{t}^{n}$ in terms of $v^{n}$. The next lemma says how.

LemMa 6.4. If $u$ is holomorphic on $D(a, \tau)$, then

$$
|u(y, t)| \leqslant C(y)(\tau-|t|)^{-\lambda}
$$

for some constant $\lambda \geqslant 0$ implies that

$$
\left|u_{t}(y, t)\right| \leqslant e(\lambda+1) C(y)(\tau-|t|)^{-\lambda-1} .
$$


Proof. For any fixed $y$ Cauchy's inequality allows one to estimate $u_{t}\left(y, t_{0}\right)$ in terms of the maximum of $u(y, t)$ on a disk of radius $(1+\lambda)^{-1}$, centred at $t_{0}$. The result is

$$
\begin{aligned}
\left|u_{t}\left(y, t_{0}\right)\right| & \leqslant\left(\frac{\tau-\left|t_{0}\right|}{1+\lambda}\right)^{-1} \sup \left\{|u(y, t)|:\left|t-t_{0}\right| \leqslant \frac{\tau-\left|t_{0}\right|}{1+\lambda}\right\} \\
& \leqslant\left(\frac{\lambda+1}{\tau-\left|t_{0}\right|}\right) C(y)\left\{\left(\frac{\lambda}{\lambda+1}\right)\left(\tau-\left|t_{0}\right|\right)\right\}^{-\lambda} \\
& \leqslant(\lambda+1)(1+1 / \lambda)^{\lambda} C(y)\left(\tau-\left|t_{0}\right|\right)^{-\lambda-1} .
\end{aligned}
$$

Since $(1+1 / \lambda)^{\lambda}<e$, this proves the lemma.

By induction we shall show that

$$
\left|v^{n}(y, t)\right| \leqslant c A_{n}|y|^{2}\left(\frac{c K|y|^{2}}{\tau-|t|}\right)^{n-1}
$$

and along the way we shall compute the constants $A_{n}$.

From Lemma 6.3 one sees that the inequality (16) certainly holds for $n=1$, if one takes $A_{1}=\|f\| / 4(\operatorname{Re}(\alpha)+1)$.

Assume that (16) holds for some $n \geqslant 1$; then by Lemma 6.4

$$
\left|v^{n}\right|+\left|v_{t}^{n}\right| \leqslant(\tau+n e) A_{n}|y|^{2 n}(\tau-|t|)^{-n},
$$

and by Lemma 6.3 this implies that

$$
\left|v^{n+1}\right| \leqslant K \frac{\tau+n e}{(n+1)(n+\operatorname{Re}(\alpha))} A_{n}|y|^{2(n+1)}(\tau-|t|)^{-n} .
$$

Thus (16) holds, if we put

$$
A_{n}=\frac{(c K)^{n}}{n !} A_{1}
$$

where

The series

$$
c:=\sup _{n \geqslant 1} \frac{\tau+n e}{n+\operatorname{Re}(\alpha)} .
$$

$$
u(y, t)=\sum_{n=1}^{\infty} v^{n}(y, t)
$$

therefore converges uniformly and absolutely on compact subsets of $D(a, \tau)$, so that the desired solution exists.

By (17) we also have

$$
\left|v^{n}\right|+\left|v_{t}^{n}\right| \leqslant\left(\frac{\tau}{n}+e\right) \frac{A_{1}|y|^{2}}{(n-1) !}\left(\frac{c K|y|^{2}}{\tau-|t|}\right)^{n-1},
$$

which shows that the solution $u$ satisfies

$$
|u(y, t)|+\left|u_{t}(y, t)\right| \leqslant c\|f\||y|^{2} \sum_{n=1}^{\infty} \frac{1}{(n-1) !}\left(\frac{c K|y|^{2}}{\tau-|t|}\right)^{n-1} .
$$

and this implies the estimate of the theorem. 
Proof of Theorem 6.1. By looking at $u(y, t)-\varphi(t)$ instead of $u$, we can assume that the initial value $\varphi(t)$ vanishes.

Given $\tau$ we shall show that there is a solution of (13) on $D\left(\rho a, \rho^{2} \tau\right)$, for some $\rho$ (in fact $\rho=\pi / \sinh (\pi)$ ), and small enough $a>0$.

Choose $r>0$ so that for some $K$

$$
\left|f_{u}\right|,\left|f_{v}\right|,\left|f_{u u}\right|,\left|f_{u v}\right|,\left|f_{v v}\right| \leqslant K
$$

for $|u|,|v| \leqslant 2 r$, and $|y| \leqslant 2 a,|t| \leqslant 2 \tau$.

Starting with $u^{0}=0$, we inductively define a sequence of holomorphic functions $u_{n}$, as follows:

$$
\begin{aligned}
e^{n} & =-\mathscr{A}_{\alpha}\left(u^{n}\right)+\mathscr{F}\left(u^{n}\right), \\
h^{n} & =\left[\mathscr{A}_{\alpha}-d \mathscr{F}\left(u^{n}\right)\right]^{-1}\left(e^{n}\right), \\
u^{n+1} & =u^{n}+h^{n} .
\end{aligned}
$$

Here $\mathscr{F}(u):=f\left(u, u_{t}, y, t\right)$, and $d \mathscr{F}(u)$ is the linear operator

$$
d \mathscr{F}(u) v=f_{v}\left(u(y, t), u_{t}(y, t), y, t\right) v_{t}+f_{u}\left(u(y, t), u_{t}(y, t), y, t\right) v,
$$

so that in (19) $h^{n}$ is defined to be the solution of

$$
\mathscr{A}_{\alpha} h^{n}-d \mathscr{F}\left(u^{n}\right) h^{n}=e^{n}(y, t), \quad h^{n}(0, t)=h_{t}^{n}(0, t)=0 .
$$

Since $u^{0}=0$, it follows that $e^{0}$ is a holomorphic function on $D(2 a, 2 \rho)$, and by Theorem $6.2, h^{0}$ will be a holomorphic function on $D(2 a, 2 \rho)$, so that on $\Omega_{0}=D(a, \rho)$ we have

$$
|h(y, t)|+\left|h_{t}(y, t)\right| \leqslant C|y|^{2} .
$$

Therefore the second function of our sequence, $u^{1}$, will satisfy $|u(y, t)|+\left|u_{t}(y, t)\right| \leqslant r$ on some smaller polydisk $\Omega_{1}=D\left(\rho_{1} a, \rho_{1}^{2} \tau\right)\left(0<\rho_{1}<1\right)$. The coefficients in (19) then will satisfy $\left|f_{u}\right|,\left|f_{v}\right| \leqslant K$ so that one can again use Theorem 6.2 to construct a solution, $h^{1}$, of (19).

Going on this way, one sees that the sequence of functions is well defined, and that their domains form a set of shrinking polydisks $\Omega_{n}=D\left(\rho_{n} a, \rho_{n}^{2} \tau\right)$.

We shall show that one can take $\rho_{0}=1$ and $\rho_{n+1}=\left(1+n^{-2}\right)^{-1} \rho_{n}$, so that the $\rho_{n}$ are bounded away from zero. In fact, one easily verifies that $\rho_{n} \downarrow \pi / \sinh (\pi)$.

This would imply that the $u^{n}$ are defined on some common $\Omega_{\rho}$. We shall also show that they converge uniformly on compact subsets of $\Omega_{\rho}$. The limit is then the solution of (13) for which we are looking.

Combining (18), (19), (20) one finds that

$$
e^{(n+1)}=\mathscr{F}\left(u^{n}\right)+d \mathscr{F}\left(u^{n}\right) h^{n}-\mathscr{F}\left(u^{n}+h^{n}\right),
$$

and by expanding $\mathscr{F}\left(u^{n}+h^{n}\right)$ in a Taylor series with remainder term, this leads to

$$
\left|e^{(n+1)}\right| \leqslant K\left|h^{n}\right|^{2} \text {. }
$$

Assume that for all $n<N$ one has

$$
\left|u^{n}\right|+\left|u_{t}^{n}\right| \leqslant r
$$

on $\Omega_{n}$, and assume that $N$ is the largest integer (or $+\infty$ ) with this property. Then, for any $n<N$, (21) has a solution on $\Omega_{n}$, which can be estimated by

$$
\left|h^{n}\right|+\left|h_{t}^{n}\right| \leqslant c\left\|e^{n}\right\|_{n}|y|^{2} \exp \left(\frac{c K|y|^{2}}{\rho_{n}^{2} \tau-|t|}\right),
$$

where $c$ is a constant which only depends on $\tau$ and $\operatorname{Re}(\alpha)$. The norm $\|f\|_{n}$ denotes the supremum norm on $\Omega_{n}$ :

$$
\|f\|_{n}=\sup _{\Omega_{n}}|f| .
$$


On the smaller polydisk $\Omega_{n+1}$ one has

Using (23) we get

$$
\begin{aligned}
\left|h^{n}\right|+\left|h_{t}^{n}\right| & \leqslant c\left\|e^{n}\right\|_{n} a^{2} \exp \left(c K \frac{\rho_{n+1}^{2}}{\rho_{n}^{2}-\rho_{n+1}^{2}}\right) \\
& =c\left\|e^{n}\right\|_{n} a^{2} e^{c n^{2} k} .
\end{aligned}
$$

$$
\left|h^{n}\right|+\left|h_{t}^{n}\right| \leqslant c K a^{2} e^{c n^{2} K}\left\|h^{n-1}\right\|_{n}^{2},
$$

which implies that for all $n \leqslant N$ one has

and hence,

$$
\left\|h^{n}\right\|_{n} \leqslant c K a^{2} e^{c n^{2} K}\left\|h^{n-1}\right\|_{n-1}^{2}
$$

$$
\begin{aligned}
\left\|h^{n}\right\|_{n} & \leqslant\left(c K a^{2}\right)^{1+2+\ldots+2^{n-1}} e^{c K\left(n^{2}+2(n-1)^{2}+\ldots+2^{n-1} 1^{2}\right.}\left\|h^{0}\right\|_{0}^{2^{n}} \\
& \leqslant\left(C a\left\|h^{0}\right\|_{0}\right)^{2^{n}},
\end{aligned}
$$

where $C$ is a constant which does not depend on $a$ or $n$. We have used that

$$
n^{2}+2(n-1)^{2}+\ldots+2^{n-1} 1^{2} \leqslant C 2^{n}
$$

for some constant $C$, and we have implicitly assumed that $a<1$.

Since $n^{2} \leqslant 2^{n}$, the estimate (25) for $|h|+\left|h_{t}\right|$ shows that

$$
\left|h^{n}\right|+\left|h_{t}^{n}\right| \leqslant\left(C a\left\|h^{0}\right\|_{0}\right)^{2^{n}}
$$

with a larger constant $C$.

By (22) we have $\left|h^{0}\right| \leqslant C|y|^{2}$, so that we can choose $a$ so small that

$$
\left\|h^{0}\right\|_{0}+\sum_{n=1}^{\infty}\left(\mathrm{Ca}\left\|h^{0}\right\|_{0}\right)^{2^{n}}<r .
$$

Then it follows from $u^{n}=h^{0}+h^{1}+\ldots+h^{n-1}$ that $N=\infty$; that is, (24) holds for all $n \geqslant 1$, and that the $u^{n}$ indeed converge uniformly on compact subsets of

$$
\Omega_{\infty}=\bigcap_{n=1}^{\infty} \Omega_{n} \text {. }
$$

This completes the proof of Theorem 6.1, and also the proof of Theorem 1.3.

\section{References}

1. S. B. ANGENENT, 'The Morse-Smale property for a semilinear parabolic equation', J. Differential Equations 82 (1986) 427-442.

2. S. B. ANGENENT, 'Analyticity of the interface of the porous media equation after the waiting time', Proc. Amer. Math. Soc. 102 (1988) 329-336.

3. S. B. ANGENENT, 'The zero set of a solution of a parabolic equation', J. Reine Angew. Math. 390 (1988) 79-96.

4. S. B. ANGENENT and B. Fiedler, 'The dynamics of rotating waves in scalar reaction diffusion equations', Trans. Amer. Math. Soc. 307 (1988) 545-568.

5. D. G. ARonson and J. L. VAZQuEz, 'Eventual regularity and asymptotic concavity for flows in one dimensional porous media', Arch. Rational Mech. Anal. 99 (1987) 329-348.

6. L. A. Caffarelli and A. FriedmanN, 'Regularity of the free boundary for the one-dimensional flow of gas in a porous medium', Amer. J. Math. 101 (1979) 1193-1218.

7. A. FriedmanN, 'On the regularity of the solutions of nonlinear elliptic and parabolic systems of partial differential equations', J. Math. Mech. 7 (1958) 43-61.

8. V. GUILLEMIN and M. GoluBITSKY, Stable mappings and their singularities, Graduate Texts in Mathematics 14 (Springer, Berlin, 1973).

9. K. Höllig and H. O. KREISS, ' $C^{\infty}$-regularity for the porous medium equation', University of Wisconsin at Madison, Computer Sciences department, Technical report \# 600 . 
10. F. John, Partial differential equations, Applied Mathematical Sciences 1 (Springer, Berlin, 1971).

11. H. Matano, 'Nonincrease of the lapnumber for a solution for a one-dimensional semi-linear parabolic equation', J. Fac. Sci. Univ. Tokyo, IA 29 (1982) 401-441.

12. C. Sturm, 'Mémoire sur une classe d'équations à différences partielles', J. Math. Pures Appl. (1836).

13. G. SzEGö, Orthogonal polynomials, 4th edition, Colloquium Publications 23 (American Mathematical Society, Providence, 1975).

University of Wisconsin - Madison

Department of Mathematics

Van Vleck Hall, 480 Lincoln Drive

Madison, Wisconsin 53706

USA 